\title{
Mamíferos do campus da Universidade Federal de Santa Maria, Rio Grande do Sul, Brasil
}

\author{
Tiago Gomes dos Santos ${ }^{1,6}$; Marcia Regina Spies ${ }^{2}$; Katia Kopp ${ }^{3}$; Rafael Trevisan ${ }^{4}$; Sonia Zanini Cechin ${ }^{5}$ \\ ${ }^{1}$ Programa de Pós-Graduação em Ciências Biológicas (Zoologia), Instituto de Biociências, \\ Universidade Estadual Paulista - UNESP, \\ Av. 24a, $n^{\circ}$ 1515, Bairro Bela Vista, CEP 13506-900, Rio Claro, SP, Brasil \\ ${ }^{2}$ Programa de Pós-Graduação em Entomologia, \\ Faculdade de Filosofia Ciências e Letras de Ribeirão Preto, Universidade de São Paulo - USP, \\ Av. Bandeirantes, 3900, CEP 14040-901, Ribeirão Preto, SP, Brasil, www.ffclrp.usp.br \\ ${ }^{3}$ Programa de Pós-Graduação em Ciências Ambientais- CIAMB, ICB 4, \\ Secretaria de Pós-Graduação em Ciências Ambientais, Campus Samambaia, \\ Universidade Federal de Goiás - UFG, CEP 74001-970, CP 131, Goiânia, GO, Brasil, www.ufg.br \\ ${ }^{4}$ Programa de Pós-Graduação em Botânica, Universidade Federal do Rio Grande do Sul, \\ Av. Bento Gonçalves, 9500, prédio 43433, sala 214, \\ CEP 91501-970, Porto Alegre, RS, Brasil, www.ufgrs.br \\ ${ }_{5}^{5}$ Departamento de Biologia, Centro de Ciências Naturais e Exatas, Universidade Federal de Santa Maria \\ Av. Roraima s/no, CEP 97105-900, Santa Maria, RS, Brasil, www.ufsm.br \\ ${ }^{6}$ Autor para correspondência: Tiago Gomes dos Santos, e-mail: frogomes@yahoo.com.br,www.rc.unesp.br
}

Santos, T. G.; Spies, M. R.; Kopp, K.; Trevisan, R.; Cechin, S. Z. Mammals of the campus of the Universidade Federal de Santa Maria, Rio Grande do Sul, Brazil. Biota Neotrop., vol. 8, no. 1, Jan./Mar. 2008. Available from: <http://www.biotaneotropica.org.br/v8n1/pt/abstract?inventory+bn00508012008>.

\begin{abstract}
The study was conducted in the Campus of the Universidade Federal de Santa Maria (UFSM), which is located in the Central Depression of Rio Grande do Sul State, in the Pampa biome. Here, a mammal list is presented and spatial occupation and conservation strategies of local mammals are discussed. Between November 2001 and October 2002, 26 native species and two exotic species of mammals (Lepus europaeus and Mus musculus) were recorded, representing 14 families. Most recorded species presents wide distribution, is likely associated to open environments and is tolerant to human disturbances. However, we also recorded three species that are considered rare or threatened in the State of Rio Grande do Sul (Lontra longicaudis, Monodelphis dimidiata and Nyctinomops laticaudatus), for which conservation strategies are recommended. The low species richness recorded in the Campus can be related to the strong pressure of human disturbances, to the small extension of the studied area or to historical factors, as the studied area is originally a grassland (Pampa), a type of environment containing a lower mammalian diversity than native forests.
\end{abstract}

Keywords: conservation, inventory, Lontra longicaudis, mammals, Pampa.

Santos, T. G.; Spies, M. R.; Kopp, K.; Trevisan, R.; Cechin, S. Z. Mamíferos do campus da Universidade Federal de Santa Maria, Rio Grande do Sul, Brasil. Biota Neotrop., vol. 8, no. 1 jan./mar. 2008. Disponível em: <http://www.biotaneotropica.org.br/v8n1/pt/abstract?inventory+bn00508012008>.

Resumo: O estudo foi desenvolvido no Campus da Universidade Federal de Santa Maria (UFSM), localizado na Depressão Central do Rio Grande do Sul, no bioma Pampa. Aqui uma listagem de mamíferos é apresentada e são discutidas a ocupação espacial e estratégias de conservação da mastofauna local. Entre novembro de 2001 e outubro de 2002 foram registradas 26 espécies nativas e duas espécies exóticas (Lepus europaeus e Mus musculus) de mamíferos, distribuídas em 14 famílias. A maioria das espécies registradas apresenta ampla distribuição, é comumente associada a áreas abertas e apresenta tolerância a distúrbios antrópicos. Entretanto, também foram registradas espécies consideradas raras ou ameaçadas no Estado do Rio Grande do Sul (Lontra longicaudis, Monodelphis dimidiata e Nyctinomops laticaudatus), para as quais são sugeridas estratégias de conservação. A baixa diversidade de espécies registrada no Campus pode estar relacionada à forte pressão de modificações antrópicas, à pequena extensão da área estudada ou a fatores históricos, já que a área de estudo é originalmente campestre (Pampa), tipo de ambiente que abriga menor diversidade de mamíferos que áreas de florestas nativas.

Palavras-chave: conservação, inventário, Lontra longicaudis, mamíferos, Pampa. 


\section{Introdução}

Atualmente são conhecidas 5.418 espécies de mamíferos, as quais apresentam grande diversificação na ocupação dos hábitats terrestres e aquáticos (Wilson \& Reeder 2005). Mais de 650 espécies ocorrem no Brasil (Reis et al. 2006) e 158 são listadas para o Rio Grande do Sul, onde 33 espécies são consideradas ameaçadas (duas espécies de Didelphimorphia, duas de Cingulata, uma de Chiroptera, duas de Primates, 13 de Carnivora, duas de Cetacea, sete de Artiodactyla, uma de Perissodactyla e três de Rodentia) (sensu Fontana et al. 2003).

Levantamentos da mastofauna do Rio Grande do Sul são escassos (Behr \& Fortes 2002), apesar dos primeiros estudos datarem do século 19 (Ihering 1893). Para a região da Quarta Colônia de Imigração Italiana foram listadas 58 espécies de mamíferos (Behr \& Fortes 2002), abrangendo oito municípios situados na Encosta Inferior do Nordeste (área de Floresta Estacional Decidual) e apenas um na Depressão Central do Estado (área de Pampa).

O Pampa constitui o bioma brasileiro com o terceiro maior número de espécies de mamíferos ameaçadas (13\%) (Costa et al. 2005) e historicamente tem sido profundamente modificado pelas atividades humanas (e.g, pastoreio excessivo, queimadas, invasão de espécies exóticas e conversão em áreas agriculturáveis), restando muitas vezes apenas pequenos remanescentes em uma paisagem predominantemente agrícola (Risser 1997, Porto 2002, Bencke 2003). Este bioma ocupa uma área de aproximadamente $700.000 \mathrm{~km}^{2}$, compartilhados entre Argentina, Brasil e Uruguai (Bilenca \& Miñarro 2004). No Estado do Rio Grande do Sul, o bioma abrange cerca de $176.000 \mathrm{~km}^{2}$, equivalendo a $63 \%$ do território gaúcho e a $2,1 \%$ do território nacional (Collares 2006). Recentemente o Pampa enfrenta a expansão da soja e dos projetos que visam o "crescimento da metade sul do Estado" através da conversão das áreas campestres em extensas áreas de plantio de árvores exóticas (principalmente Pinus e Eucalyptus), com fins de produção de celulose e madeira (Chomenko 2006).

Desta forma, o desenvolvimento de estudos de levantamentos de espécies em áreas campestres é fundamental para o fornecimento de ferramentas que subsidiem propostas de manejo e conservação do Pampa gaúcho. Estudos recentes documentaram o impacto negativo da urbanização e da conversão da vegetação nativa em lavouras sobre a fauna de répteis do Pampa da região de Santa Maria (Santos et al. 2005, Winck et al. 2007). Entretanto, o único levantamento prévio da mastofauna do Campus da Universidade Federal de Santa Maria (UFSM) foi realizado na década de 90 (Lippold et al. 1992), mas não foi publicado. No presente estudo é apresentado o inventário de mamíferos do Campus da UFSM e é discutida a ocupação espacial, bem como, estratégias de conservação da mastofauna local.

\section{Material e Métodos}

O estudo foi desenvolvido no Campus da Universidade Federal de Santa Maria (UFSM), localizado na Depressão Central do Rio Grande do Sul (29 $42^{\prime}$ S and 53 $3^{\circ} 42^{\prime} \mathrm{W}$; $95 \mathrm{~m}$ de altitude, Figura 1) no bioma Pampa (IBGE 2004). A área estudada possui cerca de $6 \mathrm{~km}^{2}$, dos quais 2,1 são urbanizados e o restante está irregularmente distribuído em uma paisagem bastante fragmentada, modificada por pastagens, lavouras e plantio de Eucalyptus spp. e Pinus spp. (ver detalhes em Santos et al. 2005).

O clima da área estudada é subtropical úmido com verões rigorosos (tipo Cfa de Köppen), caracterizado por médias térmicas entre 17 e $19{ }^{\circ} \mathrm{C}$ e chuvas bem distribuídas ao longo do ano (índices pluviométricos entre 1500 e $1750 \mathrm{~mm}$ ) (Pereira et al. 1989).

As amostragens de campo foram realizadas mensalmente e tiveram duração de quatro dias por mês, no período de novembro de 2001 a outubro de 2002. Como método de amostragem foi utilizado busca visual, na qual os diversos ambientes disponíveis na área (área urbana, campo, capoeira, lavoura, mata e margens de açudes, banhados e riachos) foram investigados. As atividades tiveram início pela tarde e se estenderam até próximo às 23:00 horas. Foram considerados registros diretos (visualização) e indiretos (fezes, odor, pegadas e restos mortais). Os quirópteros foram capturados com auxílio de puçá e manualmente durante a vistoria de fendas de dilatação e sótãos de prédios. Em quatro ocasiões foram utilizadas redes-de-neblina (tipo mist nets), durante quatro horas logo após o ocaso. O material testemunho das espécies registradas (espécimes, moldes em gesso de pegadas, fezes e restos mortais) foi incorporado à Coleção Científica de Mamíferos da UFSM (ZUFSM) e Museu de Ciências Naturais da ULBRA Canoas (MCNU), RS (Apêndice I).

Para complementação da listagem, foram utilizados os registros da Coleção Científica de Mamíferos da UFSM (Departamento de Biologia, Setor de Zoologia, ZUFSM), considerando os exemplares coletados anteriormente a este estudo no Campus e áreas adjacentes (Apêndice I). A identificação das espécies foi realizada com base em Vizotto \& Taddei (1973), Silva (1994), Bredt et al. (1996), Barquez et al. (1999), Becker \& Dalponte (1999), Voss et al. (2005), Weksler \& Bonvicino (2005) e Reis et al. (2006).

A eficiência de coleta foi avaliada pela construção da curva do coletor, utilizando 100 randomizações. O total de registros efetuados em cada mês de estudo foi considerado como uma unidade amostral ( $\mathrm{n}=12$ amostras). A análise foi realizada no programa EstimateS 7.5 (Colwell 2005).

A constância de ocorrência das espécies foi calculada pelo índice de Silveira-Neto et al. (1976), segundo o qual espécies encontradas em mais de 50\% das amostras (campanhas mensais de amostragem) são consideradas constantes; àquelas presentes entre 25 e $50 \%$ são acessórias e aquelas encontradas em menos de $25 \%$ das amostras são consideradas ocasionais. As espécies foram ainda analisadas quanto à ocupação espacial dos diversos ambientes disponíveis na área (área urbana, campo, capoeira, lavoura, mata e margens de açudes, banhados e riachos), sendo consideradas especialistas aquelas cuja freqüência de ocorrência (porcentagem do número total de registros) foi igual

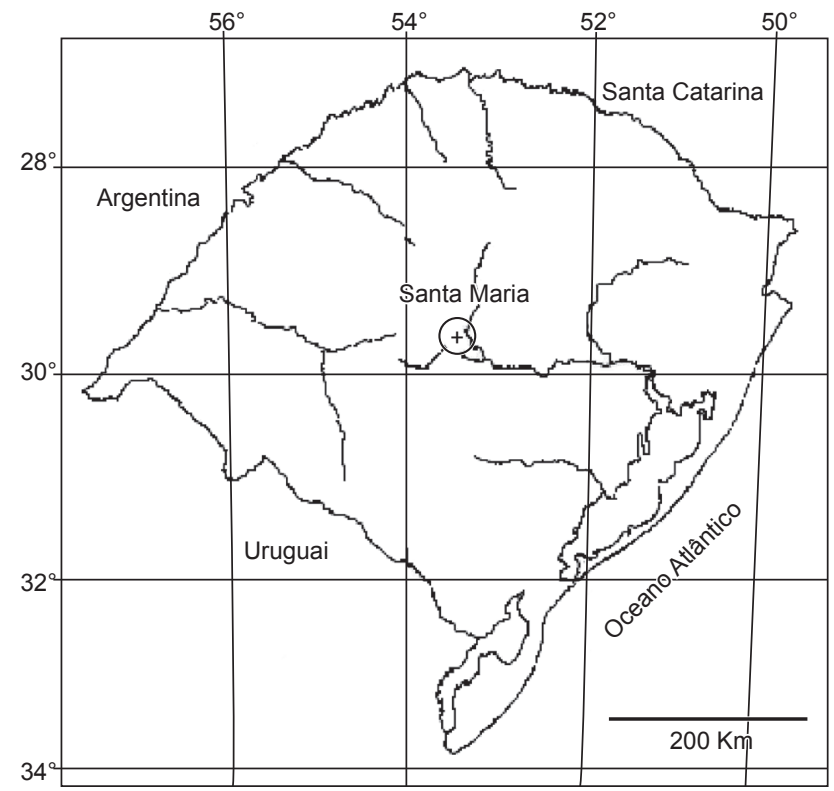

Figura 1. Mapa da localização do município de Santa Maria, Rio Grande do Sul.

Figure 1. Map of the location of Santa Maria Municipality, Rio Grande do Sul. 
ou superior a $70 \%$ em um dos ambientes estudados, e generalistas aquelas cuja frequiência de ocorrência foi inferior a 70\%. Registros de espécies provenientes da Coleção Científica foram desconsiderados para a realização das análises acima mencionadas.

\section{Resultados e Discussão}

Foram registradas 26 espécies nativas, distribuídas em 12 famílias pertencentes a cinco ordens (Tabela 1), que correspondem a $16 \%$ das 158 listadas para o Rio Grande do Sul (sensu Fontana et al. 2003). A maior riqueza de espécies foi registrada para as ordens Chiroptera e Rodentia (Tabela I), um resultado já esperado, pois estas constituem as ordens mais diversas de mamíferos (Eisenberg \& Redford 1999, Reis et al 2006).

O único representante da ordem Lagomorpha (Lepus europaeus) é uma espécie exótica de origem européia, considerada praga agrícola em vários países da América do Sul (Grigera \& Rapoport 1983, Jaksic et al. 2002). Além desta, outra espécie exótica foi registrada na área estudada, Mus musculus, um roedor comensal do Velho Mundo, que

Tabela 1. Composição taxonômica, forma de registro, índice de constância de ocorrência (IC) e ocupação espacial da mastofauna do Campus da Universidade Federal de Santa Maria, Rio Grande do Sul. Forma de registro: fezes (F), odor (O), pegadas (P), visualização (V) e restos mortais (RM); IC: acessória (A), ocasional (O) e constante (C); Ocupação espacial: área urbana (AU), campo nativo (CA), capoeira (CP), lavoura (LA), mata (MA) e margens de corpos d'água (MC), * espécies registradas por visualização em área adjacente ou por exame de Coleção Científica de Mamíferos da UFSM (ZUFSM) e Museu de Ciências Naturais da ULBRA Canoas (MCNU). Classificação conforme Wilson \& Reeder (2005).

Table 1. Taxonomic composition, type of record, index of constancy of occurrence (IC) and spatial occupation of mammals from the Campus of the Universidade Federal de Santa Maria, Rio Grande do Sul. Type of record: feaces $(\mathrm{F})$, odor $(\mathrm{O})$, footprint $(\mathrm{P})$, visualization $(\mathrm{V})$ and remains (RM); IC: accessory (A), occasional (O) and constant (C); Spatial occupation: urban area (AU), native grasslands (CA), native shrubland (CP), farmland (LA), forest (MA) and edge of water bodies (MC), * species recorded by visualization in adjacent area or by examination of specimens kept in the scientific mammal collection of the Universidade Federal de Santa Maria (ZUFSM) and Museu de Ciências Naturais da ULBRA Canoas (MCNU). Classification follows Wilson \& Reeder (2005).

\begin{tabular}{|c|c|c|c|c|c|c|c|c|}
\hline \multirow[t]{2}{*}{ Taxa } & \multirow{2}{*}{$\begin{array}{c}\text { Forma de } \\
\text { registro }\end{array}$} & \multirow[t]{2}{*}{ IC } & \multicolumn{6}{|c|}{ Ocupação espacial (\%) } \\
\hline & & & $\mathbf{A U}$ & CA & $\mathbf{C P}$ & $\mathbf{L A}$ & MA & MC \\
\hline \multicolumn{9}{|l|}{ CARNIVORA (5) } \\
\hline \multicolumn{9}{|l|}{ Canidae } \\
\hline Lycalopex gimnoscercus (Fisher, 1814)* & $\mathrm{P}, \mathrm{F}$ & A & - & - & - & 33 & 67 & - \\
\hline \multicolumn{9}{|l|}{ Mephitidae } \\
\hline Conepatus chinga (Molina, 1792) & $\mathrm{O}, \mathrm{P}$ & A & 29 & 14 & 57 & - & - & - \\
\hline \multicolumn{9}{|l|}{ Mustelidae } \\
\hline Galictis cuja (Molina, 1792) & $\mathrm{P}, \mathrm{V}$ & $\mathrm{A}$ & & - & - & - & 60 & 40 \\
\hline Lontra longicaudis (Olfers, 1818) & $\mathrm{F}, \mathrm{P}, \mathrm{V}$ & $\mathrm{C}$ & - & - & - & - & - & 100 \\
\hline \multicolumn{9}{|l|}{ Procyonidae } \\
\hline Procyon cancrivorus (Cuvier, 1798) & $\mathrm{P}, \mathrm{V}$ & A & - & 12 & 26 & - & 12 & 50 \\
\hline \multicolumn{9}{|l|}{ CHIROPTERA (7) } \\
\hline \multicolumn{9}{|l|}{ Molossidae } \\
\hline Molossus ater E. Geoffroy, 1805 & $\mathrm{~V}$ & A & 100 & - & - & - & - & - \\
\hline Nyctinomops laticaudatus (E. Geoffroy, 1805) & $\mathrm{V}$ & $\mathrm{C}$ & 100 & - & - & - & - & - \\
\hline \multicolumn{9}{|l|}{ Phyllostomidae } \\
\hline Glossophaga soricina (Pallas, 1766) & $\mathrm{V}$ & $\mathrm{O}$ & - & - & - & - & 100 & - \\
\hline \multicolumn{9}{|l|}{ Vespertilionidae } \\
\hline Epitesicus brasiliensis (Desmarest, 1819) & $\mathrm{V}$ & $\mathrm{O}$ & 100 & - & - & - & - & - \\
\hline Lasiurus borealis (Müller, 1776) & $\mathrm{V}$ & $\mathrm{O}$ & 50 & - & - & - & 50 & - \\
\hline Lasiurus ega (Gervais, 1856) & Coleção & & & & & & & \\
\hline Myotis nigricans (Schinz, 1821) & $\mathrm{V}$ & $\mathrm{O}$ & 100 & - & - & - & - & - \\
\hline \multicolumn{9}{|l|}{ CINGULATA (3) } \\
\hline \multicolumn{9}{|l|}{ Dasypodidae } \\
\hline Dasypus hybridus (Desmarest, 1804) & $\mathrm{P}, \mathrm{RM}$ & A & - & 34 & 33 & 33 & - & - \\
\hline Dasypus novemcinctus Linnaeus, 1758 & $\mathrm{P}$ & $\mathrm{O}$ & - & - & 100 & - & - & - \\
\hline Euphractus sexcinctus (Linnaeus, 1758) & $\mathrm{RM}$ & $\mathrm{O}$ & - & 100 & - & - & - & - \\
\hline \multicolumn{9}{|l|}{ DIDELPHIMORPHIA (4) } \\
\hline \multicolumn{9}{|l|}{ Didelphidae } \\
\hline Didelphis albiventris Lund, 1840 & $\mathrm{P}, \mathrm{V}$ & $\mathrm{C}$ & 13 & 13 & 29 & 2 & 5 & 38 \\
\hline Lutreolina crassicaudata (Desmarest, 1804) & $\mathrm{P}$ & $\mathrm{C}$ & 4 & - & 10 & 4 & 10 & 72 \\
\hline Monodelphis brevicaudis (Olfers, 1818) * & Coleção & & & & & & & \\
\hline Monodelphis dimidiata (Wagner, 1847) * & Coleção & & & & & & & \\
\hline \multicolumn{9}{|l|}{ LAGOMORPHA (1) } \\
\hline Leporidae & & & & & & & & \\
\hline
\end{tabular}


Tabela 1. Continuação..

\begin{tabular}{|c|c|c|c|c|c|c|c|c|}
\hline \multirow[t]{2}{*}{ Taxa } & \multirow{2}{*}{$\begin{array}{c}\text { Forma de } \\
\text { registro }\end{array}$} & \multirow[t]{2}{*}{ IC } & \multicolumn{6}{|c|}{ Ocupação espacial (\%) } \\
\hline & & & $\mathbf{A U}$ & CA & $\mathbf{C P}$ & LA & MA & MC \\
\hline Lepus europaeus Pallas 1778 & $\mathrm{~F}, \mathrm{P}, \mathrm{V}$ & $\mathrm{C}$ & 5 & 42 & 5 & 32 & 16 & - \\
\hline \multicolumn{9}{|l|}{ RODENTIA (8) } \\
\hline \multicolumn{9}{|l|}{ Caviidae } \\
\hline Cavia aperea Erxleben, 1777 & $\mathrm{~F}, \mathrm{P}, \mathrm{V}$ & $\mathrm{C}$ & - & 41 & 27 & 2 & - & 30 \\
\hline Hydrochoerus hydrochaeris (Linnaeus, 1766) & $\mathrm{F}, \mathrm{P}, \mathrm{V}$ & A & - & - & - & - & - & 100 \\
\hline \multicolumn{9}{|l|}{ Cricetidae } \\
\hline Akodon paranaensis Christoff et al., 2000* & Coleção & & & & & & & \\
\hline Calomys laucha (Fischer, 1914)* & Coleção & & & & & & & \\
\hline Nectomys squamipes (Brants, 1827) & $\mathrm{P}, \mathrm{V}$ & $\mathrm{C}$ & - & - & - & 12 & - & 88 \\
\hline Oligoryzomys nigripes (Olfers, 1818)* & Coleção & & & & & & & \\
\hline \multicolumn{9}{|l|}{ Muridae } \\
\hline Mus musculus Linnaeus, 1758 & Coleção & & & & & & & \\
\hline \multicolumn{9}{|l|}{ Myocastoridae } \\
\hline Myocastor coypus (Molina, 1782) & F, P, RM, V & $\mathrm{C}$ & - & 8 & - & - & - & 92 \\
\hline
\end{tabular}

vive essencialmente em habitações humanas, freqüentando despensas, quartos e bibliotecas (Oliveira \& Bonvicino, 2006).

A maioria das espécies nativas registradas é considerada de ampla distribuição, comumente associada a áreas abertas e tolerante a distúrbios antrópicos (e.g., Barquez et al. 1999, Eisenberg \& Redford 1999, Schneider 2000, González 2001, Cáceres et al. no prelo). Entretanto, três delas (Lontra longicaudis, Monodelphis dimidiata e Nyctinomops laticaudatus) estão listadas no Livro Vermelho da Fauna Ameaçada de Extinção do Rio Grande do Sul (Fontana et al. 2003).

Lontra longicaudis consta como espécie vulnerável no Rio Grande do Sul devido à poluição de cursos d'água e suas margens, drenagem de banhados, destruição da vegetação ripária e caça decorrente dos conflitos com pescadores e piscicultores (Indrusiak \& Eizirik 2003). Os registros de Lontra longicaudis na área presentemente estudada foram efetuados em córregos fortemente impactados por efluentes (provenientes do Campus e de áreas urbanas adjacentes), supressão da mata ciliar, canalização, erosão, assoreamento e deposição de entulhos nas margens.

Monodelphis dimidiata consta como 'Dados Insuficientes' no Rio Grande do Sul, devido à falta de informações sobre sua situação populacional (Vieira \& Iob 2003). Segundo os autores, esta espécie é encontrada no sudeste do Brasil, Uruguai e região pampeana da Argentina, ocupando áreas abertas, vegetação arbustiva, banhados e campos cultivados. A inclusão de $M$. dimidiata no presente estudo foi baseada em exemplares capturados em armadilhas de interceptação e queda (pitfalls) instaladas em área de campo nativo com presença de gado, localizada nas adjacências do Campus.

Nyctinomops laticaudatus consta como 'Dados Insuficientes' no Rio Grande do Sul, onde existem apenas três registros, efetuados em regiões floristicamente distintas (Pacheco \& Freitas 2003). Estes autores sugerem que a forte ação antrópica e descaracterização dos hábitats nativos tenham provocado a redução dos abrigos utilizados por essa espécie no Estado. Entretanto, nossos registros permitem inferir que indivíduos desta espécie são tolerantes ao processo de urbanização, pois colônias de centenas de indivíduos habitam os sótãos e as fendas de dilatação dos prédios do Campus.

A curva de acumulação de espécies apresentou formato ascendente (Figura 2), o que sugere expectativa de incremento de espécies na área estudada. De acordo com Santos (2003), em ecossistemas tropicais as curvas de acumulação de espécies raramente estabilizam.

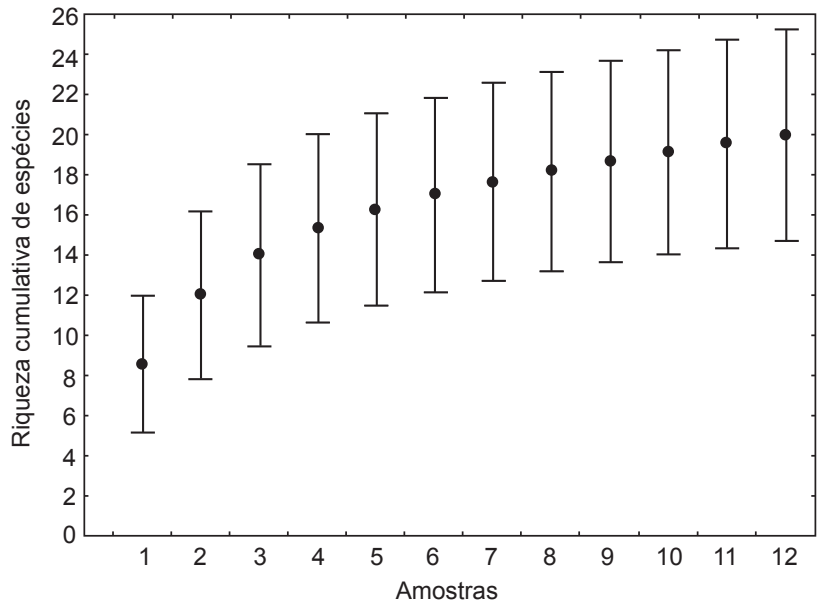

Figura 2. Curva de acumulação de espécies de mamíferos do Campus da Universidade Federal de Santa Maria, Rio Grande do Sul, ao longo de 12 meses de amostragem. Os pontos representam o número médio de espécies e as barras indicam o intervalo de confiança de $95 \%$ obtidos por randomização das amostras mensais.

Figure 2. Accumulation curve of mammal species in the Campus of the Universidade Federal de Santa Maria, Rio Grande do Sul, during 12 months of sampling. Dots represent the mean number of species and bars indicate the confidence interval of $95 \%$ obtained by randomization of monthly samples.

Entretanto, consideramos que este possível viés foi atenuado pelo exame de espécimes depositados na Coleção Científica da UFSM, que permitiu o acréscimo de sete espécies à listagem local. Para Chiroptera, a amostragem empregada privilegiou o registro de espécies insetívoras que geralmente utilizam edificações como abrigo (Bredt et al. 1996). Desta forma, o incremento de espécies não pode ser descartado com o emprego de amostragem utilizando metodologia específica para este grupo (redes-de-neblina).

Pelo menos $70 \%$ das espécies registradas no Campus da UFSM são compartilhadas com a região da Quarta Colônia de Imigração Italiana (Behr \& Fortes 2002). Este resultado também foi observado para os répteis do Campus e interpretado como indicativo de dispersão 
de elementos herpetofaunísticos comuns entre as Províncias Zoogeográficas do Planalto e do Pampa (Santos et al. 2005). No presente estudo, esta semelhança faunística pode ser explicada pela grande proximidade entre as áreas (menos de $100 \mathrm{~km}$ ); pela semelhança estrutural (presença de Pampa na região da Quarta Colônia), bem como pelo predomínio de espécies de ampla distribuição (Eisenberg \& Redford 1999, Reis et al. 2006) na composição da mastofauna do Campus, as quais tipicamente ocorrem tanto em áreas abertas como em áreas florestadas.

Duas espécies de felinos típicas de áreas campestres (Leopardus geofroy e Oncifelis colocolo) poderiam ocorrer na área estudada (González 2001, Cáceres et al. no prelo). A ausência destes registros pode ser atribuída à pequena extensão da área estudada e à forte antropização do campo nativo.

Quanto ao índice de constância, foi registrado número semelhante de espécies nas três categorias utilizadas: oito espécies constantes; sete acessórias e seis espécies ocasionais (Tabela 1). Dentre as espécies constantes, estão aquelas associadas à grande disponibilidade de corpos d'água da área estudada (Lontra longicaudis, Lutreolina crassicaudata, Nectomys squamipes e Myocastor coypus), espécies comumente associadas à zona urbana (Didelphis albiventris e Nyctinomops laticaudatus) e espécies com ampla ocorrência nos hábitats da área estudada (Lepus europaeus e Cavia aperea). Para as categorias de espécies acessórias e ocasionais, entretanto, não foi observado um conjunto claro de características comuns.

Quanto à ocupação espacial, 12 espécies (57\%) foram consideradas hábitat-especialistas (Tabela 1). Cinco destas tiveram ocorrência associada a margens de corpos d'água da área estudada (Hydrochaeris hydrochaeris, Lontra longicaudis, Lutreolina crassicaudata, Myocastor coypus e Nectomys squamipes), corroborando as informações da literatura (e.g., Silva 1994, Becker \& Dalponte 1999, Schneider 2000, Indrusiak \& Eizirik 2003, Veira \& Iob 2003). Molossus ater e Nyctinomops laticaudatus foram considerados hábitat-especialistas por utilizarem as fendas de dilatação e sótãos dos prédios como abrigo (Bredt et al. 1996). Os dados para as demais espécies consideradas especialistas na ocupação espacial (Dasypus novemcinctus, Epitesicus brasiliensis, Euphractus sexcinctus, Glossophaga soricina e Myotis nigricans) devem ser interpretados com cautela, pois estas foram ocasionais na área estudada.

O restante das espécies registradas $(9,43 \%)$ é representado por espécies generalistas quanto à ocupação espacial (Tabela 1). Para a maioria dessas espécies a plasticidade na ocupação espacial é corroborada pela literatura (e.g., Silva 1994, Becker \& Dalponte 1999, Schneider 2000, Jaksic et al. 2002). Entretanto, para Lasiurus borealis a precisão da informação aqui apresentada está comprometida pelo baixo número de registros efetuados.

\section{Conclusão}

Foram registradas, na região do Campus da UFSM, 26 espécies nativas de mamíferos, distribuídas em 12 famílias. A maioria das espécies é considerada de ampla distribuição e comumente associada a áreas abertas. A baixa diversidade de espécies registrada pode estar relacionada à forte pressão das modificações antrópicas, à pequena extensão da área estudada, bem como fatores históricos, já que o Campus, originalmente campestre (Pampa), abrigaria naturalmente menor diversidade de mamíferos que áreas florestadas. Porém, é importante ressaltar que a diversidade de mamíferos conhecida para a área estudada poderá ser incrementada com a realização de futuros estudos utilizando métodos de amostragem complementares (e.g., armadilhas fotográficas e de interceptação e queda).

O registro de uma espécie de marsupial e outra de quiróptero consideradas raras (Monodelphis dimidiata e Nyctinomops laticaudatus, respectivamente) e de uma espécie de mustelídeo (Lontra longicaudis) ameaçada de extinção no Estado, indicam a importância do Campus da UFSM como mantenedor da diversidade de mamíferos do Pampa gaúcho. Além disso, os resultados do presente estudo constituem uma base inicial de conhecimentos sobre história natural e uso de hábitat, subsidiando futuras ações conservacionistas locais. Neste contexto, considerando a forte pressão das modificações antrópicas na área estudada, recomendamos o desenvolvimento de medidas urgentes que permitam a conservação da mastofauna do Campus, em especial de Lontra longicaudis: i) desenvolvimento de projetos de educação ambiental envolvendo a comunidade universitária, bem como da zona urbana adjacente, enfocando a importância da preservação das matas ciliares, do tratamento dos esgotos e do destino adequado do lixo e dos entulhos; ii) tratamento dos efluentes liberados nos córregos, dentro e fora do Campus; iii) recuperação das matas ciliares; e iv) desenvolvimento do Zoneamento Ambiental e do Plano de Manejo do Campus.

\section{Agradecimentos}

Ao Dr. Alexandre Christoff (MCNU, ULBRA Canoas), Dr. Nilton Cáceres (UFSM) e Dr. Everton Behr (CESNORS/UFSM) pela ajuda na identificação dos roedores e marsupiais. À MSc. Josi Cerveira, ao MSc. Alberto Senra Gonçalves, à Dra. Renata Pardini e ao revisor anônimo pelas valiosas sugestões e comentários sobre o manuscrito. Às biólogas Camila Both e MSc. Gisele Winck pela ajuda nas atividades de campo. Ao Cassiano Roman e a Veridiana Spies Betat pela ajuda na organização do material-testemunho junto às Coleções de Mamíferos ZUFSM e MCNU, respectivamente.

\section{Referências Bibliográficas}

BARQUEZ, R.M., MARES, M.A. \& BRAUN, J.K. 1999. The bats of Argentina. Museum of Texas Tech University, Lubbock. 275p.

BECKER, M. \& DALPONTE, J.C. 1999. Rastros de mamíferos silvestres brasileiros: um guia de campo. Editora da Universidade de Brasília, Brasília. 180p.

BEHR, E.R. \& FORTES, V.B. 2002. Mamíferos. In Quarta Colônia: Inventários Técnicos (J. Itaqui, org.). Condesus Quarta Colônia, Santa Maria, p. 231-239.

BENCKE, G.A. 2003. Apresentação. In C.S Fontana, G.A. Bencke, R.E. Reis, eds.). Livro vermelho da fauna ameaçada de extinção no Rio Grande do Sul. EDIPUCRS, Porto Alegre, p. 14-21.

BILENCA, D.N. \& MIÑARRO, F.O. 2004. Identificación de Áreas Valiosas de Pastizal (AVPs) em las Pampas y Campos de Argentina, Uruguay y sur de Brasil. Fundación Vida Silvestre, Buenos Aires. 323p.

BREDT, A., ARAÚJO, F.A.A., CAETANO-JÚNIOR, J., RODRIGUES, M.G.R., YOSHIZAWA, M., SILVA, M.M.S., HARMANI, N.M.S., MASSUNAGA, P.N.T., BÜRER, S.P., PORTO, V.A.R. \& UIEDA, W. 1996. Morcegos em áreas urbanas e rurais: manual de manejo e controle. Fundação Nacional de Saúde, Brasília. 117p.

CACERES, N.C., CHEREM, J.J. \& GRAIPEL, M.E. no prelo. Distribuição dos mamíferos terrestres da região Sul do Brasil. Ciência \& Ambiente.

CHOMENKO, L. 2006. O Desenvolvimento na metade Sul do Rio Grande do Sul. Ecoagência, Porto Alegre. Disponível em: http://www.ecoagencia. com.br/index.php?option $=$ content $\&$ task $=$ view $\&$ id $=1687 \&$ Itemid $=62$. (Acesso em: 20/11/2006).

COLLARES, J.E.R. 2006. Mapa de biomas do Brasil. In Simpósio Mapeamento da vegetação Brasileira, $57^{\circ}$ Congresso Nacional de Botânica. Sociedade Botânica do Brasil, Gramado, p. 306-309.

COLWELL, R.K. 2005. EstimateS: Statistical estimation of species richness and shared species from samples. Version 7.5. Disponível em: http:// viceroy.eeb.unconn.edu/estimates. (Acesso em: 20/11/2006).

COSTA, L.P., LEITE, Y.L.R., MENDES, S.L. \& ALBERT, D.D. 2005. Conservação de mamíferos no Brasil. Megadiversidade 1(1):103-112. 
EISENBERG, J.F. \& REDFORD, K.H. 1999. Mammals of the Neotropics The Central Neotropics: Ecuador, Peru, Bolivia, Brazil. The University of Chicago Press, Chicago. 609p.

FONTANA, C.S., BENCKE, G.A. \& REIS, R.E. 2003. Livro vermelho da fauna ameaçada de extinção no Rio Grande do Sul. EDIPUCRS, Porto Alegre. 632p.

GONZÁLEZ, E.M. 2001. Guía de campo de los Mamíferos de Uruguay. Introducción al estudio de los mamíferos. Vida Silvestre, Sociedad Uruguaya para la Conservación de la Naturaleza. Graphis Ltda, Montevideo. $339 \mathrm{p}$.

GRIGERA, D.E. \& RAPOPORT, E.H. 1983. Status and distribution of the European hare in South America. J. Mamm. 64(1):163-166.

IBGE. 2004. Mapa de Biomas do Brasil. Primeira aproximação. Disponível em: http://www2.ibge.gov.br/download/mapas_murais/biomas_pdf.zip. (Acesso em: 12/06/2006).

IHERING, H. VON. 1893. Os Mamíferos do Rio Grande do Sul. Anuário do estado do Rio Grande do Sul 1:41-77.

INDRUSIAK, C. \& EIZIRIK, E. 2003. Carvívoros. In C.S Fontana, G.A. Bencke, R.E. Reis, eds.). Livro vermelho da fauna ameaçada de extinção no Rio Grande do Sul. EDIPUCRS, Porto Alegre, p. 507-533.

JAKSIC, F.M., IRIARTE, J.A., JIMÉNEZ, J.E. \& MARTÍNEZ, D.R. 2002. Invaders without frontiers: cross-border invasions of exotic mammals. Biol. Invas. 4:157-173.

LIPPOLD, H.O., LINK, D. \& DEPRÁ, G.T. 1992. Levantamento da fauna de mamíferos ocorrentes na área do Campus da Universidade Federal de Santa Maria, resultados preliminares. In XIX Congresso Brasileiro de Zoologia. Sociedade Brasileira de Zoologia, Belém, p. 164.

OLIVEIRA, J.A. \& BONVICINO, C.R. 2006. Ordem Rodentia. In N.R. Reis, A.L. Peracchi, W.A. Pedro, \& I.P. Lima (eds.). Mamíferos do Brasil. Imprensa da UEL, Londrina, p. 347-406.

PACHECO, S.M. \& FREITAS, T.R.O. 2003. Quirópteros. In C.S Fontana, G.A. Bencke, R.E. Reis (eds.). Livro vermelho da fauna ameaçada de extinção no Rio Grande do Sul. EDIPUCRS, Porto Alegre, p. 493-497.

PEREIRA, P.R.B., NETTO, L.R.G., BORIN, C.J.A. \& SARTORI, M.G.B. 1989. Contribuição à geografia física do município de Santa Maria: unidades de paisagem. Geogr. Ens. Pesqui. 3:37-68.

PORTO, M.L. 2002. Os Campos Sulinos: sustentabilidade e manejo. Ciência \& Ambiente, 24(4):119-138.
REIS, N.R., PERACCHI, A.L., PEDRO, W.A. \& LIMA, I.P. 2006. Mamíferos do Brasil. Imprensa da UEL, Londrina. 437p.

RISSER, P.G. 1997. Diversidade em e entre prados. In E.O. Wilson (ed.). Biodiversidade. Nova Fronteira, Rio de Janeiro, p. 224-229.

SANTOS, A.J. dos. 2003. Estimativas de riqueza em espécies. In: L.Cullen Jr., R. Rudram \& C.Valladares-Padua (orgs.). Métodos de estudos em biologia da conservação e manejo da vida silvestre. UFPR/Fundação O Boticáio, Curitiba, p. 19-41.

SANTOS, T.G., KOPP, K.A., SPIES, M.R., TREVISAN, R. \& CECHIN, S.Z. 2005. Répteis do Campus da Universidade Federal de Santa Maria, RS, Brasil. Biota Neotropica 5(1): http://www.biotaneotropica.org.br/v5n1/ pt/abstract?inventory+BN02705012005.

SCHNEIDER, M. 2000. Mastofauna. In C.J. Alho (ed.). Fauna silvestre da região do rio Manso, MT. MMA, Edições IBAMA/ELETRONORTE, Brasília, p. 217-267.

SILVA, F. 1994. Mamíferos silvestres do Rio Grande do Sul. 2.ed. Fundação Zoobotânica do Rio Grande do Sul, Porto Alegre. 244p.

SILVEIRA-NETO, S., NAKANO, O., BARBIN, D. \& NOVA, N.A.V. 1976. Manual de ecologia dos insetos. Editora Agronômica Ceres, São Paulo. 419p.

VIEIRA, E. \& IOB, G. 2003. Marsupiais. In C.S Fontana, G.A. Bencke, R.E. Reis, eds.). Livro vermelho da fauna ameaçada de extinção no Rio Grande do Sul. EDIPUCRS, Porto Alegre, p. 481-486.

VIZOTTO, L.D. \& TADDEI, V.A. 1973. Chave para determinação de quirópteros brasileiros. Bol. Ciên. Fac. Ciên. Letras São José do Rio Preto 1:1-72.

VOSS, R.S., LUNDE, D.P. \& JANSA, S.A. 2005. On the contents of Gracilinanus Gardner \& Creighton, 1989, with the description of a previously unrecognized clade of small didelphid marsupials. Amer. Mus. Novitates 3482:1-34.

WEKSLER, M. \& BONVICINO, C.R. 2005. Taxonomy of pigmy rice rats (genus Oligoryzomys, Rodentia, Sigmodontinae) of the Brazilian Cerrado, with the description of two new species. Arquivos do Museu Nacional 63(1):113-130

WILSON, D.E. \& REEDER, D.M. 2005. Mammal Species of the World. Johns Hopkins University Press, Washington. 2142p.

WINCK, G.R., SANTOS, T.G. \& CECHIN, S.Z. 2007. Snake assemblage of anthropogenic grassy fields, Rio Grande do Sul State, southern Brazil: the case of populational fluctuations of Liophis poecilogyrus and Pseudablabes agassizii. Ann. Zool. Fennici 44:321-332.

Recebido em 11/04/07

Versão reformulada recebida em 21/11/07

Publicado em 01/01/08 


\section{Apêndice 1}

Material testemunho dos mamíferos que ocorrem no Campus da Universidade Federal de Santa Maria e áreas adjacentes depositados na Coleção Científica de Mamíferos da Universidade Federal de Santa Maria (ZUFSM) e Museu de Ciências Naturais da ULBRA Canoas (MCNU), Rio Grande do Sul.

\section{Appendix 1}

Voucher material of mammals that occur in the Campus of the Universidade Federal de Santa Maria and surrounding areas deposited in the scientific collection of the Universidade Federal de Santa Maria (ZUFSM) and Museu de Ciências Naturais da ULBRA Canoas (MCNU), Rio Grande do Sul.

Material testemunho: Akodon paranaensis (MCNU 873, 874), Calomys laucha (MCNU 876), Cavia aperea (ZUFSM 0364), Conepatus chinga (ZUFSM 0360), Dasypus hybridus (ZUFSM 0368), Dasypus novemcinctus (ZUFSM 0369), Didelphis albiventris (ZUFSM 0361), Epitesicus brasiliensis (ZUFSM 0330), Euphractus sexcinctus (ZUFSM 0370), Galictis cuja (ZUFSM 0357), Glossophaga soricina (ZUFSM 0345), Hydrochoerus hydrochaeris (ZUFSM 0365), Lasiurus borealis (ZUFSM 0339, 0341), Lasiurus ega (ZUFSM 0298), Lepus europaeus (ZUFSM 0363), Lontra longicaudis (ZUFSM 0358), Lutreolina crassicaudata (ZUFSM 0362), Lycalopex gimnoscercus (ZUFSM 0356), Molossus ater (ZUFSM 0319, 0335, 0340), Monodelphis brevicaudis (ZUFSM 0360, 0361, 0362, 0365, 0366), Monodelphis dimidiata (ZUFSM 0348, 0349, 0350), Mus musculus (ZUFSM 0351, 0352), Myocastor coypus (ZUFSM 0367), Myotis nigricans (ZUFSM 0322, 0332, 0333), Nectomys squamipes (ZUFSM 0366), Nyctinomops laticaudatus (ZUFSM 0316, 0318, 0321, 0323-0329, 0334, 0347), Oligoryzomys nigripes (ZUFSM 0353-0355, MCNU 875, 877) e Procyon cancrivorus (ZUFSM 0359). 